\title{
A NUMERICAL SOLUTION OF VOLTERRA INTEGRAL-ALGEBRAIC EQUATIONS USING BERNSTEIN POLYNOMIALS
}

\author{
ELHAM ENTEGHAMI-ORIMI, AZIZOLLAH BABAKHANI, \\ AND HASSAN HOSAINZADEH
}

Received 29 May, 2019

\begin{abstract}
The principal aim of this paper is to serve the numerical solution for a linear integralalgebraic equation (IAE) by using Bernstein polynomials. Employing of Bernstein polynomials, the system of integral equations is approximated by the Gaussian quadrature formula with respect to the Legendre weight function. The proposed method reduces the system of integral equations to a system of algebraic equations that can be easily solved by any usual numerical method. Moreover, the convergence analysis of this algorithm will be shown by preparing some theorems. Several examples are included to illustrate the efficiency and accuracy of the proposed technique and also the results are compared with the different methods.
\end{abstract}

2010 Mathematics Subject Classification: 11B68; 65H10

Keywords: Bernstein polynomials, integral algebraic equations, Gauss-Legendre collocation method, approximate solutions, convergence analysis

\section{INTRODUCTION}

Integral equation is encountered in a variety of applications in many fields including optimal control systems [9,31], potential theory [13], geophysics [12], renewal theory [1], radiation [23], optimization [20], mathematical economics [30], mathematical problems of radioactive equilibrium [34], particle transport problems of astrophysics and reactor theory [22], acoustics [33], fluid mechanics [19] and etc.

Systems of Volterra integral equations with identically singular matrices in the principal part are called integral algebraic equations. Such equations and systems frequently arise in theoretical $[7,9,14,25,29,35]$ and many applied problems especially in the fields of dynamic processes in chemical reactors [17], identification of memory kernels in heat conduction [32] viscoelastic materials [15], evolution of a chemical reaction within a small cell [16] and Kirchhoff's laws. The theory of IAEs appeared from early attempts by Gear in the 1990 that determined the difficulties of these equations. He introduced the "index reduction procedure" for IAEs system in [11] similar to that in [10] for differential algebraic equations in which if the process 
is terminated, then the index is determined. This means that under suitable conditions, there is a solution for the resulting regular system of integral equations.

Several authors have investigated the existence, uniqueness and numerical analysis of IAEs systems. Bulatov et al. [6], gave the existence and uniqueness results of solution for IAE systems with convolution kernels and defined the index in analogy to Gear's approach. Kauthen [18] applied the polynomial spline collocation method for a semi-explicit IAEs with index-1 and established global convergence as well as local super convergence. Furthermore, Brunner [4] defined the index-1 tractable for a semi-explicit form of IAEs and investigated the existence of a unique solution for these types of systems.

In this article, we study numerical methods for solving the system of Volterra integral equations of the first and second kind. More precisely, we consider the integral algebraic equation

$$
\mathbf{A}(t) \mathbf{X}(t)=\mathbf{F}(t)+\int_{0}^{t} \mathbf{K}(t, s) \mathbf{X}(s) d s, \quad 0 \leq t \leq 1
$$

where

$$
\begin{aligned}
& \mathbf{A}(t)=\left[a_{i j}(t)\right], \quad i, j=1,2, \cdots, p, \quad \mathbf{F}(t)=\left[y_{1}(t) y_{2}(t) \cdots y_{m}(t)\right]^{T}, \\
& \mathbf{K}(t, s)=\left[k_{i j}(t, s)\right], \quad i, j=1,2, \cdots, p
\end{aligned}
$$

are given functions and $\mathbf{X}(t)=\left[x_{1}(t) x_{2}(t) \cdots x_{m}(t)\right]^{T}$ is the solution to be determined. If det $\mathbf{A}(t)=0$, this system is called as Volterra Integral-Algebraic Equations (IAEs). Under the condition det $\mathbf{A}(t)=0$, the system can have several solutions or no solution at all. We apply the following theorem for the existence of the solution of (1.1).

Theorem 1 ([2]). Assume that system (1.1) with $\operatorname{det} \boldsymbol{A}(s)=0$ satisfies the following conditions:

1. $\mathbf{A}(t) \in C_{[0,1]}^{1}, \mathbf{X}(t) \in C_{[0,1]}^{1}$ and $\mathbf{K}(t, s) \in C_{\triangle}^{1}$, where $\triangle=\{0 \leq s \leq t \leq 1\}$.

2. $\operatorname{Rank} \mathbf{A}(t)=\operatorname{deg}(\operatorname{det}[\lambda \mathbf{A}(t)+\mathbf{K}(t, t)])=k$, for all $t \in[0,1]$, where $k$ is constant and $\lambda$ is a scalar.

3. $\operatorname{Rank} \mathbf{A}(0)=\operatorname{Rank}[\mathbf{A}(0) \mid \mathbf{F}(0)]$.

Then the system has a unique continuous solution.

Several researchers have adopted different techniques for solving system of integral algebraic equations [2, 5, 8, 21, 24, 28]. Our suggestion is by employing of Bernstein polynomials and combining with some other concepts to obtain a numerical solution for (1.1).

The paper is organized in the following way. In Section 2, we present the definition of the Bernstein polynomials and some fundamental properties are studied. In Section 3, we apply the Bernstein polynomials for solving system (1.1). A convergence analysis is presented in Section 4. We offer some numerical examples to illustrate the efficiency of these algorithms in Section 5. 


\section{Preliminaries}

Some necessary preliminaries and notations of Bernstein polynomials are given below which are usable later.

Definition 1 ([27]). The Bernstein polynomials of degree $n$ defined on the interval $[0,1]$ are given by

$$
B_{n, k}(t)=\left(\begin{array}{l}
n \\
k
\end{array}\right) t^{k}(1-t)^{n-k}, \quad k=0,1,2, \cdots, n,
$$

where

$$
\left(\begin{array}{l}
n \\
k
\end{array}\right)=\frac{n !}{k !(n-k) !} \quad n \in \mathbb{N} .
$$

It should be note that each of these $n+1$ polynomials having degree $n$ and satisfies the following properties:

(i) $B_{n, k}(t)=0, \quad$ if $k<0$ or $k>n$.

(ii) $B_{n, k}(0)=B_{n, k}(1)=0$, for $1 \leq k \leq n-1$.

(iii) $\sum_{k=0}^{n} B_{n, k}(t)=1$.

The Bernstein's approximation, $B_{n}(f)$ to a function $f:[0,1] \rightarrow \mathbb{R}$ is the polynomial

$$
B_{n}(f)=\sum_{k=0}^{n} f\left(\frac{k}{n}\right) B_{n, k}(t),
$$

where $B_{n, k}$ is a polynomial of degree $n$, which is defined by (2.1).

Bernstein in [3] used this approximation to give the first constructive proof of the Weierstrass theorem.

Theorem 2 ([26]). For all functions $f$ in $C[0,1]$, the sequence $B_{n}(f), n=1,2,3, \cdots$ converges uniformly to $f$, where $B_{n}(f)$ is defined by (2.2).

Suppose that $H=L^{2}[0,1]$ is a Hilbert space with the inner product defined by $\langle f, h\rangle=\int_{0}^{1} f(t) h(t)^{T} d t$ and $V=\operatorname{Span}\left\{B_{n, 0}(t), B_{n, 1}(t), \cdots, B_{n, n}(t)\right\}$. Since $V$ is a finite-dimensional subspace of $H$, it is closed and convex. Thus, for $f \in H$, there exists a unique best approximation out of $V$ such as $g$, such that [27]

$$
\|f-g\| \leq\|f-h\|, \quad \forall h \in V .
$$

Since $g \in V$, there exists a unique coefficients $c_{0}, c_{1}, \cdots, c_{n}$ such that

$$
f \simeq g=\sum_{k=0}^{n} c_{k} B_{n, k}(t)=\mathbf{C}^{T} \psi(t),
$$

where

$$
\psi^{T}=\left[B_{n, 0}, B_{n, 1}, \cdots, B_{n, n}\right] \quad \text { and } \quad \mathbf{C}^{T}=\left[c_{0}, c_{1}, \cdots, c_{n}\right] .
$$

Then, $\mathbf{C}^{T}$ can be obtained by

$$
\mathbf{C}^{T}=\mathbf{D}^{-1}\langle f, \psi\rangle,
$$


where

$$
\langle f, \psi\rangle=\int_{0}^{1} f(t) \psi(t)^{T} d t,
$$

and $\mathbf{D}$ is an $(n+1) \times(n+1)$ matrix which is said the dual matrix of $\psi(t)$ and will be introduced in the form

$$
\mathbf{D}=\langle\psi, \psi\rangle=\int_{0}^{1} \psi(t) \psi(t)^{T} d t
$$

\section{EMPLOYING OF BERNSTEIN POLYNOMIALS}

Any unknown vector-valued function $\mathbf{X}$ can be expand in the Bernstein basis as

$$
\begin{gathered}
B_{n}(\mathbf{X}(\mathbf{t}))=\left[B_{n}\left(x_{1}(t)\right), B_{n}\left(x_{2}(t)\right), \cdots, B_{n}\left(x_{m}(t)\right)\right]^{T}, \\
B_{n}\left(x_{j}(t)\right)=\sum_{k=0}^{n} c_{j, k} B_{n, k}(t), \quad t \in[0,1], j=1,2, \cdots, p,
\end{gathered}
$$

where $n$ is chosen as any positive integer and $B_{n, k}(t), k=0,1, \cdots, n$ are the Bernstein polynomials defined in the previous section.

In order to approximate the solution of (1.1), we consider the system of linear Volterra integral algebraic equations with variable coefficients in the following form:

$$
\sum_{j=1}^{p} a_{i j}(t) x_{j}(t)=f_{i}(t)+\sum_{j=1}^{p} \int_{0}^{t} k_{i j}(t, s) x_{j}(s) d s, \quad i=1,2, \cdots, p, \quad 0 \leq t \leq 1 .
$$

Substituting (3.2) in (3.3), we obtain

$$
\sum_{j=1}^{p} a_{i j}(t) B_{n}\left(x_{j}(t)\right)=f_{i}(t)+\sum_{j=1}^{p} \int_{0}^{t} k_{i j}(t, s) B_{n}\left(x_{j}(s)\right) d s, \quad i=1,2, \cdots, p .
$$

Putting (3.2) in (3.4) we have

$$
\sum_{j=1}^{p} a_{i j}(t) \sum_{k=0}^{n} c_{j, k} B_{n, k}(t)=f_{i}(t)+\sum_{j=1}^{p} \int_{0}^{t} k_{i j}(t, s) \sum_{k=0}^{n} c_{j, k} B_{n, k}(s) d s \quad i=1,2, \cdots, p .
$$

Then we get

$$
\sum_{j=1}^{p} \sum_{k=0}^{n}\left(a_{i j}(t) B_{n, k}(t)-\int_{0}^{t} k_{i j}(t, s) B_{n, k}(s) d s\right) c_{j, k}=f_{i}(t), \quad i=1,2, \cdots, p .
$$

In order to find $c_{j, k}, j=1,2, \cdots, p$ and $k=0,1,2, \cdots, n$, we convert the above equation to a linear system of equations by collocating (3.6) in $(n+1)$ Newton-Cotes collocation points $t_{l}=\frac{2 l-1}{2(n+1)}, l=0,1, \cdots, n$ on the interval $[0,1]$.

Thus we have

$$
\sum_{j=1}^{p} \sum_{k=0}^{n}\left(a_{i j}\left(t_{l}\right) B_{n, k}\left(t_{l}\right)-\int_{0}^{t_{l}} k_{i j}\left(t_{l}, s\right) B_{n, k}(s) d s\right) c_{j, k}=f_{i}\left(t_{l}\right) .
$$


In our approach, we use the quadrature rule to approximate the integrals involved in this system, which achieves zero error integration for polynomial integrands of degree less than or equal to $2 n+2$ with $n+1$ Legendre-Gauss nodes. For this purpose, a simple linear transformation must be made. So, the intervals $\left[0, t_{l}\right], l=0,1, \cdots, n$ are transferred to a fixed interval $[-1,1]$ by means of the transformation

$$
s=\frac{t_{l}}{2}+\frac{t_{l}}{2} \tau \quad \text { and } \quad d s=\frac{t_{l}}{2} d \tau
$$

Hence, employing this transformation on the second term in the left hand side of (3.7) the results is

$$
\sum_{j=1}^{p} \sum_{k=0}^{n}\left(a_{i j}\left(t_{l}\right) B_{n, k}\left(t_{l}\right)-\frac{t_{l}}{2} \int_{-1}^{1} k_{i j}\left(t_{l}, \frac{t_{l}}{2}+\frac{t_{l}}{2} \tau\right) B_{n, k}\left(\frac{t_{l}}{2}+\frac{t_{l}}{2} \tau\right) d \tau\right) c_{j, k}=f_{i}\left(t_{l}\right) .
$$

Applying the Gaussian quadrature, system (3.8) is converted to

$$
\sum_{j=1}^{p} \sum_{k=0}^{n}\left(a_{i j}\left(t_{l}\right) B_{n, k}\left(t_{l}\right)-\frac{t_{l}}{2} \sum_{r=0}^{n} \omega_{r} k_{i j}\left(t_{l}, \frac{t_{l}}{2}+\frac{t_{l}}{2} \tau_{r}\right) B_{n, k}\left(\frac{t_{l}}{2}+\frac{t_{l}}{2} \tau_{r}\right)\right) c_{j, k}=f_{i}\left(t_{l}\right),
$$

where $\tau_{r}, r=0,1, \cdots, n$ are the $n+1$ Legendre-Gauss nodes and $\omega_{r}, r=0,1, \cdots, n$ are the weights on $[-1,1]$.

For brevity, we define below symbols as:

$$
T_{l, k}^{(i, j)}=a_{i j}\left(t_{l}\right) B_{n, k}\left(t_{l}\right), \quad R_{l, k}^{(i, j)}=\frac{t_{l}}{2} \sum_{r=0}^{n} \omega_{r} k_{i j}\left(t_{l}, \frac{t_{l}}{2}+\frac{t_{l}}{2} \tau_{r}\right) B_{n, k}\left(\frac{t_{l}}{2}+\frac{t_{l}}{2} \tau_{r}\right) .
$$

Now we can write system (3.9) in the form

$$
\sum_{j=1}^{p} \sum_{k=0}^{n}\left(T_{l, k}^{(i, j)}-R_{l, k}^{(i, j)}\right) c_{j, k}=f_{i}\left(t_{l}\right), \quad i=1,2, \cdots, p, \quad l=0,1, \cdots, n .
$$

Consequently, expression (3.10) can be summarized in a matrix form as follows:

$$
\mathcal{A} x=\mathcal{Y},
$$

where

$$
\begin{aligned}
& \mathcal{X}=\left[c_{1,0}, \cdots, c_{1, n}, \cdots, c_{j, 0}, \cdots, c_{j, n}, \cdots, c_{p, 0}, \cdots, c_{p, n}\right]^{T}, \\
& \mathcal{Y}=\left[f_{1}\left(t_{0}\right), \cdots, f_{1}\left(t_{n}\right), \cdots, f_{i}\left(t_{0}\right), \cdots, f_{i}\left(t_{n}\right), \cdots, f_{p}\left(t_{0}\right), \cdots, f_{p}\left(t_{n}\right)\right]^{T},
\end{aligned}
$$

and

$$
\mathcal{A}=\left[\begin{array}{ccc}
\mathcal{A}^{(1,1)} & \cdots & \mathcal{A}^{(1, p)} \\
\vdots & \ddots & \vdots \\
\mathcal{A}^{(p, 1)} & \cdots & \mathcal{A}^{(p, p)}
\end{array}\right]
$$


Parochial matrix $\mathcal{A}^{(i, j)}$ for $(i, j)=(1,2, \cdots, p)$ is defined by

$$
\mathcal{A}^{(i, j)}=\left[\begin{array}{ccc}
\mathcal{A}_{0,0}^{(i, j)} & \cdots & \mathcal{A}_{0, n}^{(i, j)} \\
\vdots & \ddots & \vdots \\
\mathcal{A}_{n, 0}^{(i, j)} & \cdots & \mathcal{A}_{n, n}^{(i, j)}
\end{array}\right],
$$

where

$$
\mathcal{A}_{l, k}^{(i, j)}=T_{l, k}^{(i, j)}-R_{l, k}^{(i, j)} .
$$

The resulting generalized linear system can be solved for $c_{j, k}$ for $j=1,2, \cdots, p$ and $k=0,1, \cdots, n$ by a standard method, and hence $x_{j}(t)$ is obtained.

\section{CONVERGENCE ANALYSis}

In this section we proceed to prove that the present numerical method converges to the exact solutions of system (1.1). For this purpose, let us consider the Banach space $C=C[0,1], \times \cdots \times C[0,1]$ ( $p$ times) endowed with the norm

$$
\|\mathbf{X}(t)\|=\max _{1 \leq j \leq p} \max _{0 \leq t \leq 1}\left|x_{j}(t)\right| .
$$

Theorem 3. Consider the system of Volterra integral-algebraic equations (1.1). Assume that $\bar{B}_{n}(\mathbf{X}(t))$ is the best approximation of $V=\operatorname{Span}\left\{B_{n, 0}(t), B_{n, 1}(t)\right.$, $\left.\cdots, B_{n, n}(t)\right\}$ and $\hat{B}_{n}(\mathbf{X}(t))$ is the approximation solution of (1.1). Then

$$
\left\|\bar{B}_{n}(\mathbf{X}(t))-\hat{B}_{n}(\mathbf{X}(t))\right\| \leq \max _{1 \leq j \leq p} \max _{0 \leq k \leq n}\left|\bar{c}_{j, k}-\hat{c}_{j, k}\right|,
$$

where

$$
\bar{B}_{n}(\mathbf{X}(t))=\left[\sum_{k=0}^{n} \bar{c}_{1, k} B_{n, k}(t), \sum_{k=0}^{n} \bar{c}_{2, k} B_{n, k}(t), \cdots, \sum_{k=0}^{n} \bar{c}_{p, k} B_{n, k}(t)\right]^{T}
$$

and

$$
\hat{B}_{n}(\mathbf{X}(t))=\left[\sum_{k=0}^{n} \hat{c}_{1, k} B_{n, k}(t), \sum_{k=0}^{n} \hat{c}_{2, k} B_{n, k}(t), \cdots, \sum_{k=0}^{n} \hat{c}_{p, k} B_{n, k}(t)\right]^{T},
$$

where $\bar{c}_{j, k}, j=1,2, \cdots, p, k=0,1,2, \cdots, n$ is defined by $(2.3)$ and $\hat{c}_{j, k}, j=1,2, \cdots, p$, $k=0,1,2, \cdots, n$ is the solution of (3.11) computed through our presented method.

Proof. We can write

$$
\begin{aligned}
\left\|\bar{B}_{n}(\mathbf{X}(t))-\hat{B}_{n}(\mathbf{X}(t))\right\| & =\max _{1 \leq j \leq p} \max _{0 \leq t \leq 1}\left|\sum_{k=0}^{n}\left(\bar{c}_{j, k}-\hat{c}_{j, k}\right) B_{n, k}(t)\right| \\
& \leq \max _{1 \leq j \leq p} \max _{0 \leq t \leq 1} \max _{0 \leq k \leq n}\left|\bar{c}_{j, k}-\hat{c}_{j, k}\right| \sum_{k=0}^{n} B_{n, k}(t),
\end{aligned}
$$


where

$$
\sum_{k=0}^{n} B_{n, k}(t)=1, \quad 0 \leq t \leq 1
$$

This implies that

$$
\left\|\bar{B}_{n}(\mathbf{X}(t))-\hat{B}_{n}(\mathbf{X}(t))\right\| \leq \max _{1 \leq j \leq p} \max _{0 \leq k \leq n}\left|\bar{c}_{j, k}-\hat{c}_{j, k}\right|,
$$

and the proof is completed.

Now we will study the convergence of our considered method for the system of (1.1).

Theorem 4. Considering the assumptions in Theorem (3) for (1.1), let $\mathbf{F} \in C([0,1]$, $\left.\mathbb{R}^{p}\right)$ and $\mathbf{K} \in C\left([0,1] \times[0,1], \mathbb{R}^{p}\right)$. Moreover, assume that there are positive numbers $M_{1}$ and $M_{2}$ where $\|\mathbf{A}(t)\| \leq M_{1}$ and $\|\mathbf{K}(t, s)\| \leq M_{2}$ such that $M_{1}-M_{2}>0$. If $\hat{B}_{n}(\mathbf{X}(t))$ is the approximation solution of (1.1), then for sufficiently large $n$ we have

$$
\left\|\mathbf{X}(t)-\hat{B}_{n}(\mathbf{X}(t))\right\| \leq \frac{M_{1}+M_{2}}{M_{1}-M_{2}} \max _{1 \leq j \leq p} \max _{0 \leq k \leq n}\left|\bar{c}_{j, k}-\hat{c}_{j, k}\right|,
$$

Proof. The system of Volterra integral-algebraic equation (1.1) can be written as

$$
\mathbf{A}(t) \mathbf{X}(t)=\mathbf{F}(t)+\int_{0}^{t} \mathbf{K}(t, s) \mathbf{X}(s) d s
$$

Substituting $\hat{B}_{n}(\mathbf{X}(t))$ in this system yields

$$
\mathbf{A}(t) \hat{B}_{n}(\mathbf{X}(t))=\hat{\mathbf{F}}(t)+\int_{0}^{t} \mathbf{K}(t, s) \hat{B}_{n}(\mathbf{X}(s)) d s .
$$

Since $\bar{B}_{n}(\mathbf{X}(t))$ is the best approximation, we have

$$
\mathbf{A}(t) \bar{B}_{n}(\mathbf{X}(t))=\mathbf{F}(t)+\int_{0}^{t} \mathbf{K}(t, s) \bar{B}_{n}(\mathbf{X}(s)) d s .
$$

Subtracting (4.5) from (4.6), we get

$$
\mathbf{A}(t)\left(\mathbf{X}(t)-\hat{B}_{n}(\mathbf{X}(t))\right)=\mathbf{F}(t)-\hat{\mathbf{F}}(t)+\int_{0}^{t} \mathbf{K}(t, s)\left(\mathbf{X}(s)-\hat{B}_{n}(\mathbf{X}(s))\right),
$$

hence

$$
\|\mathbf{A}(t)\|\left\|\mathbf{X}(t)-\hat{B}_{n}(\mathbf{X}(t))\right\| \leq\|\mathbf{F}(t)-\hat{\mathbf{F}}(t)\|+\|\mathbf{K}(t, s)\|\left\|\mathbf{X}(s)-\hat{B}_{n}(\mathbf{X}(s))\right\|,
$$

or

$$
(\|\mathbf{A}(t)\|-\|\mathbf{K}(t, s)\|)\left\|\mathbf{X}(t)-\hat{B}_{n}(\mathbf{X}(t))\right\| \leq\|\mathbf{F}(t)-\hat{\mathbf{F}}(t)\|,
$$

therefore, we can write

$$
\left\|\mathbf{X}(t)-\hat{B}_{n}(\mathbf{X}(t))\right\| \leq \frac{1}{M_{1}-M_{2}}\|\mathbf{F}(t)-\hat{\mathbf{F}}(t)\| .
$$


In order to find a bound on $\|\mathbf{F}(t)-\hat{\mathbf{F}}(t)\|$, subtracting (4.6) from (4.7), one obtains

$$
\mathbf{F}(t)-\hat{\mathbf{F}}(t)=\mathbf{A}(t)\left(\bar{B}_{n}(\mathbf{X}(t))-\hat{B}_{n}(\mathbf{X}(t))\right)-\int_{0}^{t} \mathbf{K}(t, s)\left(\bar{B}_{n}(\mathbf{X}(s))-\hat{B}_{n}(\mathbf{X}(s))\right) d s .
$$

Then, we get

$$
\begin{aligned}
\|\mathbf{F}(t)-\hat{\mathbf{F}}(t)\| & \leq\|\mathbf{A}(t)\|\left\|\bar{B}_{n}(\mathbf{X}(t))-\hat{B}_{n}(\mathbf{X}(t))\right\|+\|\mathbf{K}(t, s)\|\left\|\bar{B}_{n}(\mathbf{X}(s))-\hat{B}_{n}(\mathbf{X}(s))\right\| \\
& \leq(\|\mathbf{A}(t)\|+\|\mathbf{K}(t, s)\|)\left\|\bar{B}_{n}(\mathbf{X}(t))-\hat{B}_{n}(\mathbf{X}(t))\right\| \\
& \leq\left(M_{1}+M_{2}\right)\left\|\bar{B}_{n}(\mathbf{X}(t))-\hat{B}_{n}(\mathbf{X}(t))\right\| .
\end{aligned}
$$

According to Theorem 3, one can write

$$
\|\mathbf{F}(t)-\hat{\mathbf{F}}(t)\| \leq\left(M_{1}+M_{2}\right) \max _{1 \leq j \leq m 0 \leq k \leq n} \max _{1 \leq j}\left|\bar{c}_{j, k}-\hat{c}_{j, k}\right| .
$$

Substituting this bound in (4.11), the purpose will be achieved.

\section{NUMERICAL EXAMPLES}

In this section some examples using our considered numerical solution are given for (1.1) to show accuracy and efficiency of this method. Exact solution of these examples are available. Comparing exact solution with approximate solution shows that this method is very accurate. A comparison of our proposed method with some other known methods has also performed. We use maximum absolute errors for selected points, defined as

$$
\left\|E_{j}(t)\right\|_{\infty}=\max \left|x_{j}(t)-\hat{x}_{j}(t)\right|, \quad j=1,2,
$$

where $x_{j}(t)$ and $\hat{x}_{j}(t)$ denotes the exact and approximate solution, respectively. It should be noted that all of the numerical computations have been performed on computer using a program written in MATLAB.

Example 1. Consider the linear Volterra integral-algebraic equations

$$
\left[\begin{array}{ll}
1 & 0 \\
0 & 0
\end{array}\right]\left[\begin{array}{l}
y(t) \\
z(t)
\end{array}\right]=\left[\begin{array}{l}
f_{1}(t) \\
f_{2}(t)
\end{array}\right]+\int_{0}^{t}\left[\begin{array}{cc}
1+t-s & e^{t-s} \\
1 & e^{2 t-s}
\end{array}\right]\left[\begin{array}{l}
y(s) \\
z(s)
\end{array}\right] d s
$$

Here

$$
\begin{aligned}
& f_{1}(t)=3 t e-t-2 e^{-t}-t+\frac{1}{2} \cos t-\frac{1}{2} \sin t-\frac{1}{2} e^{t}+1, \\
& f_{2}(t)=e^{-t}(t+1)+\frac{1}{2} e^{t} \cos t-\frac{1}{2} e^{t} \sin t-\frac{1}{2} e^{2 t}-1,
\end{aligned}
$$

with the exact solution $y(t)=t e^{-t}, z(t)=\cos t$. 
Let us check that this system satisfies the conditions required in Theorem 1. It is clear that

$$
A(t)=\left[\begin{array}{ll}
1 & 0 \\
0 & 0
\end{array}\right] \text { and } \mathbf{K}(t, s)=\left[\begin{array}{cc}
1+t-s & e^{t-s} \\
1 & e^{2 t-s}
\end{array}\right]
$$

satisfy the first condition required in Theorem 1.

On other hand $\operatorname{Rank} \mathbf{A}(t)=1$ and

$$
\lambda \mathbf{A}(t)+\mathbf{K}(t, t)=\left[\begin{array}{ll}
\lambda & 0 \\
0 & 0
\end{array}\right]+\left[\begin{array}{cc}
1 & 1 \\
1 & e^{t}
\end{array}\right]=\left[\begin{array}{cc}
\lambda+1 & 1 \\
1 & e^{t}
\end{array}\right] .
$$

Hence

$$
\left|\begin{array}{cc}
\lambda+1 & 1 \\
1 & e^{t}
\end{array}\right|=(\lambda+1) e^{t}-1 \Rightarrow \operatorname{deg}[\operatorname{det}(\lambda \mathbf{A}(t)+\mathbf{K}(t, t))]=1
$$

and

Therefore

$$
\mathbf{A}(0) \mid \mathbf{F}(0)=\left[\begin{array}{ll|l}
1 & 0 & 1 \\
0 & 0 & 0
\end{array}\right]
$$

$$
\operatorname{Rank} \mathbf{A}(0)=\operatorname{Rank}[\mathbf{A}(0) \mid \mathbf{F}(0)]=1 .
$$

Thus, this example satisfies the conditions required in Theorem 1 and therefore it has a unique continuous solution on $[0,1]$.

TABLE 1. Absolute errors of $y(t)$ and $z(t)$ at the selected points for Example 1

\begin{tabular}{|c|cc|cc|}
\hline \multirow{2}{*}{$t$} & \multicolumn{2}{|c|}{$n=8$} & \multicolumn{2}{c|}{$n=11$} \\
\cline { 2 - 5 } & $e_{1}(t)$ & $e_{2}(t)$ & $e_{1}(t)$ & $e_{2}(t)$ \\
\hline 0 & $2.6411 \mathrm{e}-09$ & $2.2546 \mathrm{e}-09$ & $1.6636 \mathrm{e}-13$ & $7.2164 \mathrm{e}-14$ \\
\hline 0.1 & $1.7224 \mathrm{e}-10$ & $2.4399 \mathrm{e}-10$ & $3.6499 \mathrm{e}-15$ & $3.8858 \mathrm{e}-14$ \\
\hline 0.2 & $4.8195 \mathrm{e}-11$ & $3.8055 \mathrm{e}-11$ & $5.5511 \mathrm{e}-16$ & $1.1435 \mathrm{e}-14$ \\
\hline 0.3 & $1.6081 \mathrm{e}-11$ & $4.5866 \mathrm{e}-11$ & $1.2490 \mathrm{e}-15$ & $1.2879 \mathrm{e}-14$ \\
\hline 0.4 & $3.5780 \mathrm{e}-11$ & $4.8837 \mathrm{e}-11$ & $1.5543 \mathrm{e}-15$ & $5.3291 \mathrm{e}-15$ \\
\hline 0.5 & $4.2929 \mathrm{e}-11$ & $2.7642 \mathrm{e}-11$ & $1.6653 \mathrm{e}-15$ & $7.3275 \mathrm{e}-15$ \\
\hline 0.6 & $5.0793 \mathrm{e}-11$ & $4.4997 \mathrm{e}-11$ & $2.2204 \mathrm{e}-15$ & $4.9960 \mathrm{e}-15$ \\
\hline 0.7 & $7.3545 \mathrm{e}-11$ & $1.0888 \mathrm{e}-11$ & $2.6645 \mathrm{e}-15$ & $1.0436 \mathrm{e}-14$ \\
\hline 0.8 & $4.8906 \mathrm{e}-11$ & $5.5758 \mathrm{e}-11$ & $3.6637 \mathrm{e}-15$ & $2.6090 \mathrm{e}-14$ \\
\hline 0.9 & $2.5692 \mathrm{e}-10$ & $6.0195 \mathrm{e}-11$ & $1.4988 \mathrm{e}-15$ & $2.2415 \mathrm{e}-13$ \\
\hline 1 & $2.1918 \mathrm{e}-09$ & $4.7665 \mathrm{e}-09$ & $1.1147 \mathrm{e}-13$ & $9.2568 \mathrm{e}-12$ \\
\hline
\end{tabular}

In this example Table 1 shows absolute errors for $n=8$ and $n=11$ by using the present method at selected points. In Table 2, we compare the maximum absolute error for the present method at $n=11$ with method [21] and method [8]. Figure 1 
TABLE 2. Comparison of the maximum absolute error for Example 1

\begin{tabular}{|c|c|c|c|}
\hline Error & Present method & Method of [21] & Method of [8] \\
\hline$E_{1}(t)$ & $1.6636 \mathrm{e}-13$ & $3 / 0473 e-05$ & $4.51 \mathrm{e}-03$ \\
\hline$E_{2}(t)$ & $9.2568 \mathrm{e}-12$ & $9 / 9757 \mathrm{e}-03$ & $4.20 \mathrm{e}-03$ \\
\hline
\end{tabular}

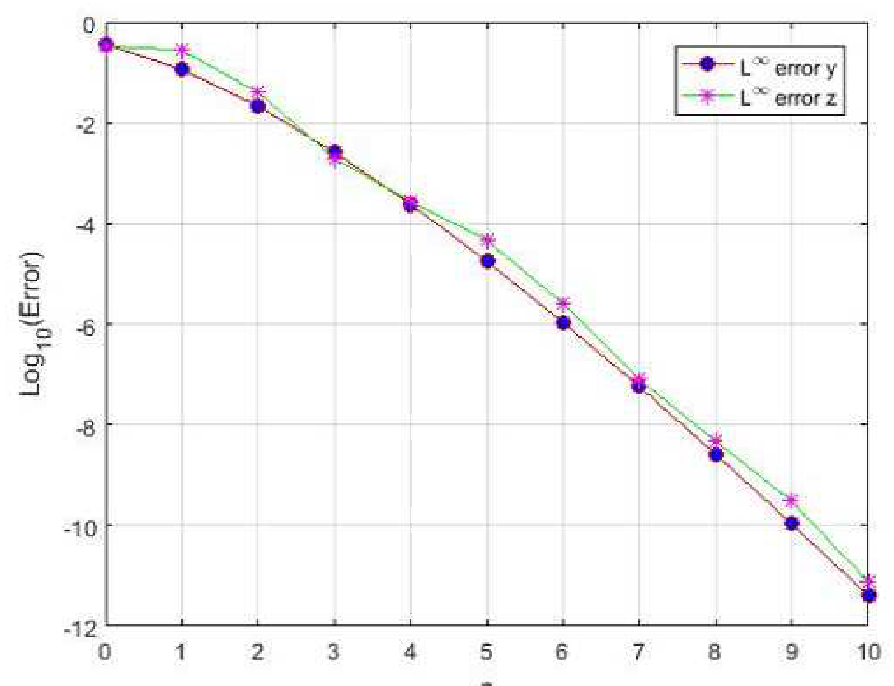

FIGURE 1. Maximum absolute error for Example 1

contains the numerical comparison of errors of our solutions using the present method for different values of $n$.

Example 2. Consider the linear Volterra integral-algebraic equations

$$
\left[\begin{array}{cc}
1 & t \\
t & t^{2}
\end{array}\right]\left[\begin{array}{l}
y(t) \\
z(t)
\end{array}\right]=\left[\begin{array}{c}
e^{t}(t+1)+t e^{-t} \\
2 t e^{t}+1+e^{-t}\left(t^{2}-1\right)
\end{array}\right]+\int_{0}^{t}\left[\begin{array}{cc}
-e^{t-s} & 0 \\
-e^{t+s} & -e^{-2 s}
\end{array}\right]\left[\begin{array}{l}
y(s) \\
z(s)
\end{array}\right] d s,
$$

with the exact solution $y(t)=e^{t}$ and $z(t)=e^{-t}$.

In Table 3, we show the absolute errors for $n=8$ and $n=11$ by using the present method. In Table 4, we compare the maximum absolute error for the present method at $n=11$ with method [2], method [5] and method [24]. Figure 2 contains the numerical comparison of errors of our solutions using the present method for different values of $n$. 
TABLE 3. Absolute errors of $y(t)$ and $z(t)$ at the selected points for Example2

\begin{tabular}{|c|cc|cc|}
\hline \multirow{2}{*}{$t$} & \multicolumn{2}{|c|}{$n=8$} & \multicolumn{2}{c|}{$n=11$} \\
\cline { 2 - 5 } & $e_{1}(t)$ & $e_{2}(t)$ & $e_{1}(t)$ & $e_{2}(t)$ \\
\hline 0 & $7.7345 \mathrm{e}-10$ & $5.9966 \mathrm{e}-10$ & $2.7700 \mathrm{e}-13$ & $2.9332 \mathrm{e}-13$ \\
\hline 0.1 & $7.2120 \mathrm{e}-11$ & $6.5572 \mathrm{e}-11$ & $9.5479 \mathrm{e}-15$ & $6.3283 \mathrm{e}-15$ \\
\hline 0.2 & $5.6464 \mathrm{e}-12$ & $1.7860 \mathrm{e}-11$ & $4.2188 \mathrm{e}-15$ & $9.1038 \mathrm{e}-15$ \\
\hline 0.3 & $1.7329 \mathrm{e}-11$ & $1.1736 \mathrm{e}-11$ & $3.1086 \mathrm{e}-15$ & $1.9984 \mathrm{e}-15$ \\
\hline 0.4 & $1.4032 \mathrm{e}-11$ & $9.9728 \mathrm{e}-12$ & $3.7748 \mathrm{e}-15$ & $2.1094 \mathrm{e}-15$ \\
\hline 0.5 & $1.3195 \mathrm{e}-11$ & $5.2208 \mathrm{e}-12$ & $3.9968 \mathrm{e}-15$ & $1.7764 \mathrm{e}-15$ \\
\hline 0.6 & $1.3600 \mathrm{e}-11$ & $4.5389 \mathrm{e}-12$ & $4.6629 \mathrm{e}-15$ & $3.1086 \mathrm{e}-15$ \\
\hline 0.7 & $9.7931 \mathrm{e}-12$ & $2.7638 \mathrm{e}-12$ & $1.3323 \mathrm{e}-15$ & $2.2204 \mathrm{e}-15$ \\
\hline 0.8 & $1.8867 \mathrm{e}-11$ & $1.2439 \mathrm{e}-12$ & $4.4409 \mathrm{e}-15$ & $2.8866 \mathrm{e}-15$ \\
\hline 0.9 & $3.5453 \mathrm{e}-11$ & $8.9101 \mathrm{e}-13$ & $8.4377 \mathrm{e}-15$ & $2.5424 \mathrm{e}-14$ \\
\hline 1 & $9.7940 \mathrm{e}-10$ & $3.3398 \mathrm{e}-10$ & $9.9210 \mathrm{e}-13$ & $1.4697 \mathrm{e}-12$ \\
\hline
\end{tabular}

TABLE 4. Comparison of the maximum absolute error for Example 2

\begin{tabular}{|c|c|c|c|c|}
\hline \multirow[t]{2}{*}{ Error } & Present method & method [2] & method [5] & method [24] \\
\hline & $n=11$ & $m=32$ & $n=10$ & $n=5 m=10$ \\
\hline$e_{1}(t)$ & $9.9210 \mathrm{e}-13$ & $2.83 \mathrm{e}-02$ & $1.10 \mathrm{e}-04$ & $1.68 \mathrm{e}-12$ \\
\hline$e_{2}(t)$ & $1.4697 \mathrm{e}-12$ & $2.34 \mathrm{e}-02$ & $1.10 \mathrm{e}-04$ & $1.48 \mathrm{e}-12$ \\
\hline
\end{tabular}

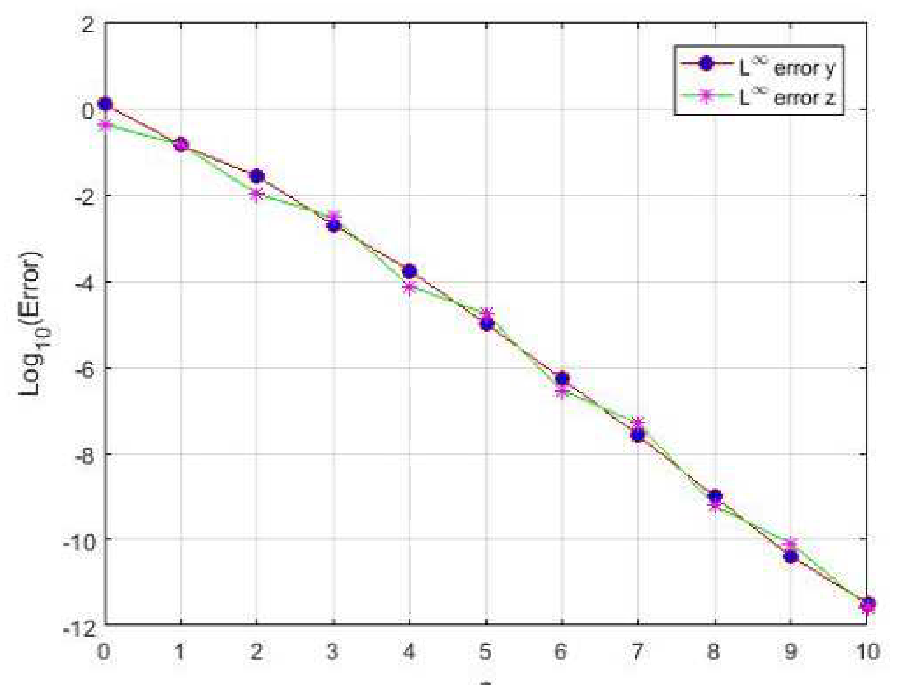

FIGURE 2. Maximum absolute error for Example 2 
Example 3. As a final example, consider the linear Volterra integral-algebraic equations

$$
\left[\begin{array}{ll}
1 & 0 \\
0 & 0
\end{array}\right]\left[\begin{array}{l}
y(t) \\
z(t)
\end{array}\right]=\left[\begin{array}{l}
f_{1}(t) \\
f_{2}(t)
\end{array}\right]+\int_{0}^{t}\left[\begin{array}{cc}
s+t & s^{2}+t^{2} \\
s-t^{2} & s+t+1
\end{array}\right]\left[\begin{array}{l}
y(s) \\
z(s)
\end{array}\right] d s
$$

where

$$
\begin{aligned}
& f_{1}(t)=-t-2(\sin t) t^{2}+2 \sin t \\
& f_{2}(t)=t^{2}-2 \sin t+t \cos t-t^{2} \cos t+1-\cos t-2 t \sin t,
\end{aligned}
$$

with the exact solution $y(t)=\sin t$ and $z(t)=\cos t$.

In Table 5, we show the absolute errors for $n=8$ and $n=11$ by using the present method. In Table 6, we compare the maximum absolute error for the present method with $n=11$ and method [28]. Figure 3 contains the numerical comparison of errors of our solutions using the present method for different values of $n$.

TABLE 5. Absolute errors of $y(t)$ and $z(t)$ at the selected points for Example 3

\begin{tabular}{|c|cc|cc|}
\hline \multirow{2}{*}{$t$} & \multicolumn{2}{|c|}{$n=8$} & \multicolumn{2}{c|}{$n=11$} \\
\cline { 2 - 5 } & $e_{1}(t)$ & $e_{2}(t)$ & $e_{1}(t)$ & $e_{2}(t)$ \\
\hline 0 & $4.3709 \mathrm{e}-10$ & $3.0667 \mathrm{e}-11$ & $3.0552 \mathrm{e}-14$ & $0.0000 \mathrm{e}+00$ \\
\hline 0.1 & $2.9435 \mathrm{e}-11$ & $3.2249 \mathrm{e}-12$ & $7.7716 \mathrm{e}-16$ & $6.3283 \mathrm{e}-15$ \\
\hline 0.2 & $6.7472 \mathrm{e}-12$ & $3.8103 \mathrm{e}-13$ & $1.1102 \mathrm{e}-16$ & $2.8866 \mathrm{e}-15$ \\
\hline 0.3 & $8.3311 \mathrm{e}-13$ & $2.9913 \mathrm{e}-12$ & $1.1102 \mathrm{e}-16$ & $2.2204 \mathrm{e}-16$ \\
\hline 0.4 & $3.6615 \mathrm{e}-12$ & $1.8513 \mathrm{e}-12$ & $1.6653 \mathrm{e}-16$ & $1.1102 \mathrm{e}-15$ \\
\hline 0.5 & $4.5935 \mathrm{e}-12$ & $3.5471 \mathrm{e}-12$ & $1.6653 \mathrm{e}-16$ & $0.0000 \mathrm{e}+00$ \\
\hline 0.6 & $5.7888 \mathrm{e}-12$ & $2.5377 \mathrm{e}-12$ & $2.2204 \mathrm{e}-16$ & $9.9920 \mathrm{e}-16$ \\
\hline 0.7 & $1.0052 \mathrm{e}-11$ & $4.9851 \mathrm{e}-12$ & $5.5511 \mathrm{e}-16$ & $2.9976 \mathrm{e}-15$ \\
\hline 0.8 & $6.6127 \mathrm{e}-12$ & $3.8752 \mathrm{e}-12$ & $8.8818 \mathrm{e}-16$ & $4.1078 \mathrm{e}-15$ \\
\hline 0.9 & $4.4303 \mathrm{e}-11$ & $8.9651 \mathrm{e}-12$ & $1.4433 \mathrm{e}-15$ & $2.2982 \mathrm{e}-14$ \\
\hline 1 & $3.6263 \mathrm{e}-10$ & $7.9842 \mathrm{e}-10$ & $1.3434 \mathrm{e}-14$ & $8.5454 \mathrm{e}-13$ \\
\hline
\end{tabular}

TABLE 6. Comparison of the maximum absolute error for Example 3

\begin{tabular}{|lclc|}
\hline Error & Present method & & method [28] \\
\cline { 2 - 2 } & $n=11$ & & $m=32$ \\
\hline$e_{1}(t)$ & $3.0552 \mathrm{e}-14$ & & $7.6 \mathrm{e}-13$ \\
\hline$e_{2}(t)$ & $8.5454 \mathrm{e}-13$ & & $1.4 \mathrm{e}-11$ \\
\hline
\end{tabular}




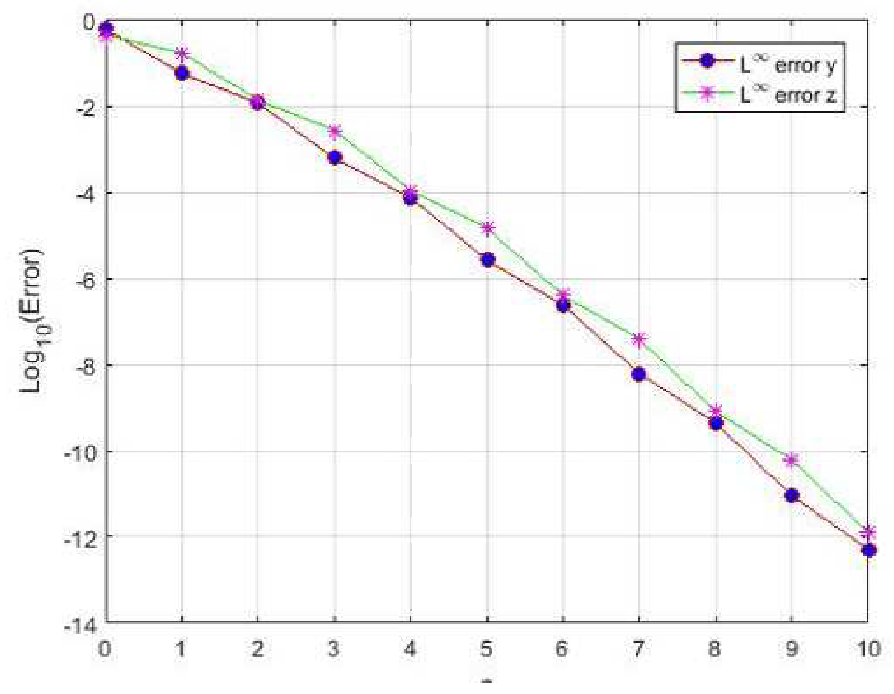

FIGURE 3. Maximum absolute error for Example 3

\section{CONCLUSION}

The aim of this manuscript was to suggest the employing of Bernstein polynomials to obtain a numerical solution for a Volterra integral-algebraic equations. The process of our considered method is that the system of integral equations has been reduced to a system of algebraic equations. In this methods, the system of integral equations was approximated by the Gauss quadrature formula with respect to the Legendre weight function and then the convergence of the proposed method was discussed. Several numerical example were presented to show that the superiority and efficiency of our numerical solution respect to some other well known methods.

\section{ACKNOWLEDGEMENT}

The authors would like to thank to the anonymous referees for their valuable comments and suggestions.

\section{REFERENCES}

[1] M. Annunziato, H. Brunner, and E. Messina, "Asymptotic stability of solutions to Volterrarenewal integral equations with space maps," Journal of Mathematical Analysis and Applications, vol. 395, no. 2, pp. 766-775, 2012, doi: 10.1016/j.jmaa.2012.05.080.

[2] V. Balakumar and K. Murugesan, "Numerical solution of Volterra integral-algebraic equations using block pulse functions," Applied Mathematics and Computation, vol. 263, pp. 165-170, 2015, doi: 10.1016/j.amc.2015.04.035.

[3] S. Bernstein, "Demonstration du theoreme de Weierstrass fondee sur le calcul des probabilites," Math. Kharkov, vol. 13, no. 1, pp. 1-2, 1912. 
[4] H. Brunner, Collocation methods for Volterra integral and related functional differential equations. Cambridge University Press, 2004, vol. 15.

[5] O. S. Budnikova and M. V. Bulatov, "Numerical solution of integral-algebraic equations for multistep methods," Computational Mathematics and Mathematical Physics, vol. 52, no. 5, pp. 691701, 2012, doi: 10.1134/S0965542512050041.

[6] M. V. Bulatov and V. F. Chistyakov, "The properties of differential-algebraic systems and their integral analogs," 1997.

[7] P. De Angelis, R. De Marchis, and A. L. Martire, "A new numerical method for a class of Volterra and Fredholm integral equations," Journal of Computational and Applied Mathematics, vol. 379, p. 112944, 2020, doi: 10.1016/j.cam.2020.112944.

[8] M. Farahani and M. Hadizadeh, "Direct regularization for system of integral-algebraic equations of index-1," Inverse Problems in Science and Engineering, vol. 26, no. 5, pp. 728-743, 2018, doi: 10.1080/17415977.2017.1347169.

[9] R. Ganji, H. Jafari, and S. Nemati, "A new approach for solving integro-differential equations of variable order," Journal of Computational and Applied Mathematics, vol. 379, p. 112946, 2020, doi: 10.1016/j.cam.2020.112946.

[10] C. W. Gear, "Differential-algebraic equation index transformations," SIAM Journal on Scientific and Statistical Computing, vol. 9, no. 1, pp. 39-47, 1988, doi: 10.1137/0909004.

[11] C. W. Gear, "Differential algebraic equations, indices, and integral algebraic equations," SIAM Journal on Numerical Analysis, vol. 27, no. 6, pp. 1527-1534, 1990, doi: 10.1137/0727089.

[12] M. Hvoždara and P. Kaikkonen, "An integral equations solution of the forward DC geoelectric problem for a 3-d body of inhomogeneous conductivity buried in a halfspace," Journal of Applied Geophysics, vol. 39, no. 2, pp. 95-107, 1998, doi: 10.1016/S0926-9851(98)00007-X.

[13] W. Hwang, "A regularized boundary integral method in potential theory," Computer Methods in Applied Mechanics and Engineering, vol. 259, pp. 123-129, 2013, doi: 10.1016/j.cma.2013.02.005.

[14] H. Jafari, H. Tajadodi, and R. M. Ganji, "A numerical approach for solving variable order differential equations based on Bernstein polynomials," Computational and Mathematical Methods, vol. 1, no. 5, p. e1055, 2019, doi: 10.1002/cmm4.1055.

[15] J. Janno and L. Von Wolfersdorf, "Inverse problems for identification of memory kernels in viscoelasticity," Mathematical methods in the applied sciences, vol. 20, no. 4, pp. 291-314, 1997, doi: 10.1002/(SICI)1099-1476(19970310)20:4;291::AID-MMA860;3.0.CO;2-W.

[16] B. Jumarhon, W. Lamb, S. McKee, and T. Tang, "A Volterra integral type method for solving a class of nonlinear initial-boundary value problems," Numerical Methods for Partial Differential Equations: An International Journal, vol. 12, no. 2, pp. 265-281, 1996, doi: 10.1002/(SICI)10982426(199603)12:2;265::AID-NUM8;3.0.CO;2-O.

[17] V. Kafarov, B. Mayorga, and C. Dallos, "Mathematical method for analysis of dynamic processes in chemical reactors," Chemical engineering science, vol. 54, no. 20, pp. 4669-4678, 1999, doi: 10.1016/S0009-2509(99)00277-8.

[18] J.-P. Kauthen, "The numerical solution of integral-algebraic equations of index 1 by polynomial spline collocation methods," Mathematics of Computation, vol. 70, no. 236, pp. 1503-1514, 2001.

[19] E. Ladopoulos, "Non-linear multidimensional singular integral equations in two-dimensional fluid mechanics," International journal of non-linear mechanics, vol. 35, no. 4, pp. 701-708, 2000, doi: 10.1016/S0020-7462(99)00052-9.

[20] S.-W. Lee, M. Yoon, and S. Cho, "Isogeometric topological shape optimization using dual evolution with boundary integral equation and level sets," Computer-Aided Design, vol. 82, pp. 88-99, 2017, doi: 10.1016/j.cad.2016.08.004.

[21] H. Liang and H. Brunner, "Integral-algebraic equations: theory of collocation methods II," SIAM Journal on Numerical Analysis, vol. 54, no. 4, pp. 2640-2663, 2016, doi: 10.1137/15M1049300. 
[22] O. Martin, "On the homotopy analysis method for solving a particle transport equation," Applied Mathematical Modelling, vol. 37, no. 6, pp. 3959-3967, 2013, doi: 10.1016/j.apm.2012.08.023.

[23] P. Martin and S. G. L. Smith, "Internal gravity waves, boundary integral equations and radiation conditions," Wave Motion, vol. 49, no. 3, pp. 427-444, 2012, doi: 10.1016/j.wavemoti.2012.01.001.

[24] F. Mirzaee, "Bernoulli collocation method with residual correction for solving integral-algebraic equations," Journal of Linear and Topological Algebra (JLTA), vol. 4, no. 03, pp. 193-208, 2015.

[25] G. R. Moallem, H. Jafari, and A. R. Adem, "A numerical scheme to solve variable order diffusionwave equations," Thermal Science, no. 00, pp. 371-371, 2019, doi: 10.2298/TSCI190729371M.

[26] M. J. D. Powell et al., Approximation theory and methods. Cambridge University Press, 1981.

[27] T. J. Rivlin, An introduction to the approximation of functions. Courier Corporation, 1981.

[28] B. Shiri, "Galerkin method for linear integral-algebraic equations of index 1," arXiv preprint arXiv:1212.3802, 2012.

[29] H. Song, Y. Xiao, and M. Chen, "Collocation methods for third-kind volterra integral equations with proportional delays," Applied Mathematics and Computation, vol. 388, p. 125509, 2021, doi: 10.1016/j.amc.2020.125509.

[30] M. Tabata, N. Eshima, Y. Sakai, and I. Takagi, “An extension of Krugman's core-periphery model to the case of a continuous domain: Existence and uniqueness of solutions of a system of nonlinear integral equations in spatial economics," Nonlinear Analysis: Real World Applications, vol. 14, no. 6, pp. 2116-2132, 2013, doi: 10.1016/j.nonrwa.2013.04.001.

[31] X. Tang, "Efficient Chebyshev collocation methods for solving optimal control problems governed by Volterra integral equations," Applied Mathematics and Computation, vol. 269, pp. 118-128, 2015, doi: 10.1016/j.amc.2015.07.055.

[32] L. Von Wolfersdorf, "On identification of memory kernels in linear theory of heat conduction," Mathematical methods in the applied sciences, vol. 17, no. 12, pp. 919-932, 1994, doi: 10.1002/mma.1670171202.

[33] X. Wang, H. Chen, and J. Zhang, "An efficient boundary integral equation method for multifrequency acoustics analysis," Engineering Analysis with Boundary Elements, vol. 61, pp. 282 286, 2015, doi: 10.1016/j.enganabound.2015.08.006.

[34] C.-Y. Wu and M.-F. Hou, "Integral equation solutions based on exact ray paths for radiative transfer in a participating medium with formulated refractive index," International journal of heat and mass transfer, vol. 55, no. 23-24, pp. 6600-6608, 2012, doi: 10.1016/j.ijheatmasstransfer.2012.06.068.

[35] Z. Xiao-yong, "A new strategy for the numerical solution of nonlinear Volterra integral equations with vanishing delays," Applied Mathematics and Computation, vol. 365, p. 124608, 2020, doi: 10.1016/j.amc.2019.124608.

\section{Authors' addresses}

Elham Enteghami-Orimi

University of Mazandaran, Faculty of Mathematical Sciences, 47416-95447 Babolsar, Iran

E-mail address: e.enteghamiestu.umz.ac.ir

\section{Azizollah Babakhani}

(Corresponding author) Babol Noshirvani University of Technology, Department of Mathematics, 4714871167 Babol, Iran

E-mail address: babakhanienit.ac.ir 


\section{Hassan Hosainzadeh}

University of Mazandaran, Faculty of Mathematical Sciences, 47416-95447 Babolsar, Iran

E-mail address: hosinzadeh99@gmail.com 